\title{
Isolation, Identification and Resistance Pattern of Microorganisms Associated with Mastitis in Buffalo
}

\author{
Ali AzamTalukder*, Hasna Hena Rahman, S. M. Jamil Mahmud, Fahmida Alam and Shuvra Kanti Dey \\ Department of Microbiology, Jahangirnagar University, Dhaka 1342, Bangladesh
}

\begin{abstract}
Prevalence of subclinical mastitis along with the etiological agents and their antimicrobial susceptibility were studied around the city of Dhaka, the capital of Bangladesh. Milk samples from 30 apparently healthy buffaloes were subjected to microbiological examinations while subclinical mastitis was diagnosed based on California Mastitis Test (CTM). Seventy percent of the buffaloes examined were positive for subclinical mastitis. A total of 42 isolates of 5 different species were identified. Among them, Staphylococcus spp. was the most frequently identified bacterial species accounting for $50 \%$ of all the isolates, followed by E.coli $(28.57 \%)$, Enterobacter (14.29\%), Bacillus spp. (4.76\%) and Proteus spp. (2.38\%). Highest resistance was found against amoxicillin (21.43\%) where chloramphenicol and ciprofloxac in proved to be a more successful antimicrobial agent, resistance against which wasonly $4.76 \%$. Results from the present study suggest that contagious mastitis controlpractice along with good personal hygiene practice is required for mastitis control in the area.
\end{abstract}

Keywords: Mastitis, Buffalo, Bangladesh, Pathogen and Staphylococcus

\section{Introduction}

In recent years, the Asian buffalo has attracted global concern because of the increasing demand of animal protein in the world. Likewise, among the livestock in Bangladesh, buffaloes play an important role in domestic economy and trade and have the potentiality to be used as a tool for poverty reduction. Therefore buffalo is considered a multipurpose animal and after cattle they provide the major contribution of the livestock sector to rural economy of Bangladesh ${ }^{1}$. Nevertheless, the research on buffalo has been much neglected in comparison to research on cattle ${ }^{2}$.

Infectious diseases are thought to be the major cause that hinders the development of livestock population including buffaloes in Bangladesh $^{3}$. Among the various infectious diseases, mastitis is considered to be one of the most important health problems in dairy cattle including buffaloes. Mastitis is defined as an inflammation of the mammary glands resulting from introduction and multiplication of pathogenic microorganisms in the gland. Mastitis not only effect on milk production but also has deleterious effects on the chemical and cytological composition of the milk. Although the causes of mastitis are mostly infectious bacteria, rarely chemical or physical agents can also be involved ${ }^{4}$. The main causative bacteria include Staphylococcus aureus, Streptococcusagalactiae, coliforms, Streptococci and Enterococci ${ }^{4}$.

Mastitis is a common disease in buffalo whose prevention and control largely depends on good management practices. To control mastitis, it is essential to work together among exposure to microbes, buffalo defense mechanisms and environmental risk factors. These are influenced by proper management practices ${ }^{5}$. Because of the small herd sizes like other developing countries, in Bangladesh, the animals are predominantly hand-milked. This increases the possibility of transmission of the infectious agent from infected to uninfected animals through milker'shand as milk is often used as a lubricant for milking. Considering the facts, the present study was designed to investigate the incidence of mastitis disease in buffalo prevailing around Dhaka in order to develop appropriate control and preventive measures against the disease.

\section{Methods and Materials}

Sample collection

A total of 30-buffaloes were randomly selected from the three distinct areas of Dhaka, the capital of Bangladesh, in farm basis, in order to find out the epidemiology of clinical and sub-clinical mastitis caused by microorganisms in the study area. During sample collection, milk samples were collected by standard milk sampling technique as described previously ${ }^{6}$, while clinical and subclinical mastitis was detected using CMT. Approximately 10 $\mathrm{ml}$ of milk samples were collected into sterile test tube after discarding the first three milking streams. A septically collected sample were placed in ice boxes and transported to the laboratory with maintaining the appropriate storage and transport temperature and other physical conditions ensuring the maximum survival of the microorganisms in the milk sample for future analysis.

\section{Isolation and identification of bacteria from milk}

In the laboratory, stored samples were kept at room temperature for 30 minutes before streaking on culture plates. Collected samples were diluted when necessary. One hundred microliter of samples was streaked on various selective agar media including, MacConkey and blood agar plates for bacterial culture and isolation. Pure colonies were identified and analyzed further morphologically and biochemically for strain identification as shown below.

*Corresponding Author:

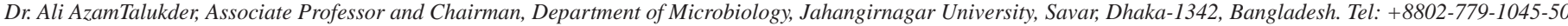
(Ext.1566), Fax: +8802-779-1052. E-mail: aat@juniv.edu tunubj@yahoo.com. 


\section{Antimicrobial susceptibility test}

The antimicrobial susceptibility of the test isolates was determined in vitro by using the standardized agar-disc-diffusion method known as the Kirby Bauer method according to Clinical and Laboratory Standards Institute (CLSI) guidelines ${ }^{7}$. Total ten antibiotic discs were used in this study, namely, amoxicillin (AML), azithromycin (AZM), chloramphenicol (CHL), ciprofloxacin (CIP), ceftriaxone (CRO), erythromycin (ERM), gentamycin (GNT), levofloxacin (LEV), nitrofurantoine (NFT) and thrimethoprim (SXT).

\section{Results and Discussion}

-Sample collection

The samples were collected during the period of September 2012 to September 2013 with the view to isolate, identify and characterize the bacterial pathogens from mastitis cases in selected areas of Bangladesh. Samples were collected from the three distinct areas of, Dhaka, Bangladesh. For the isolation and identification of the causative agent of mastitis, a total of 30 milk samples were collected from buffalo. A typical traditional (hand milking) and modern milking systems are shown in Figure 1.
Sample screening morphologically and biochemically

Morphological screening was carried out after samples were grown in different selected agar medium as shown in Figure 2. In nutrient agar plate different types of colonies were present representing different microorganisms. Mannitol Salt agar (MSA) plate was used to differentiate Mannitol fermenting Staphylococcus spp. which gave yellow colonies whereas white colonies were the indication of mannitol non-fermenting species of Staphylococcus spp. MacConkey agar plates were used for preliminary confirmation of lactose fermenting $E$. coli which gave pink colonies as shown in Figure 2C. Gram stain of MSA isolates appeared as cocci in clusters and retained violet color after gram reaction which represented that they were Gram positive cocci and gram stained MacConkey isolates; appeared rod shaped in chains and retained red colour after Gram reaction indicating Gram negative bacillus spp. (Figure 2 bottom panel).

Various isolates causing mastitis in buffalo were screened biochemically as shown in Figure 3, 4. Moreover, several sugar fermentation tests were used including Sucrose, Dextrose, Galactose, Lactose, Maltose and D-mannitol to investigate the sugar fermentation abilities of isolates (Figure 3C).

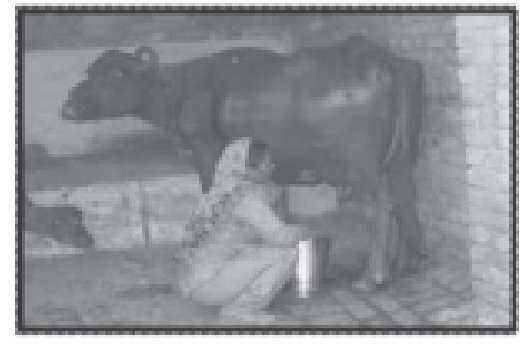

A

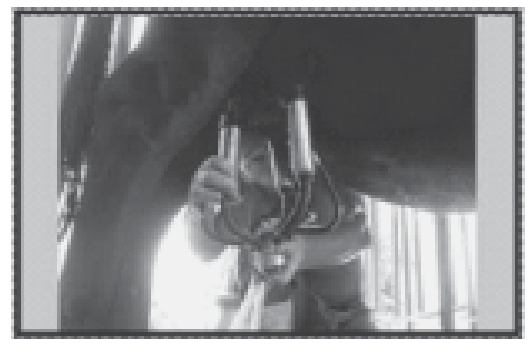

B

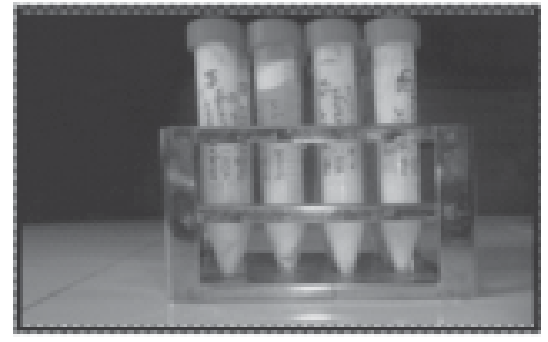

$\mathrm{C}$

Figure 1. Various types of milk Sample collection processes. (A) Traditional milking practice in Bangladesh; (B)Modern milking practice in developed countries and (C) Milk sample collected from the diseased buffalo from different areas of Bangladesh.

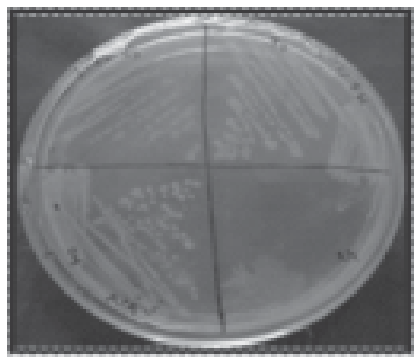

A

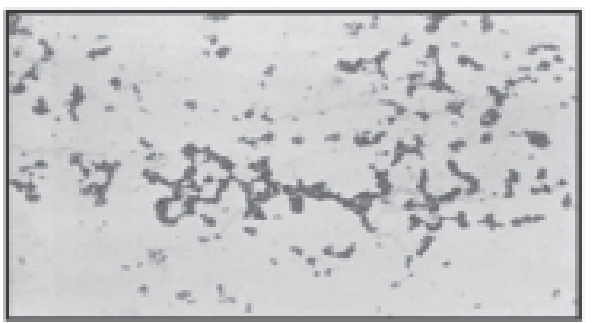

$\mathrm{D}$

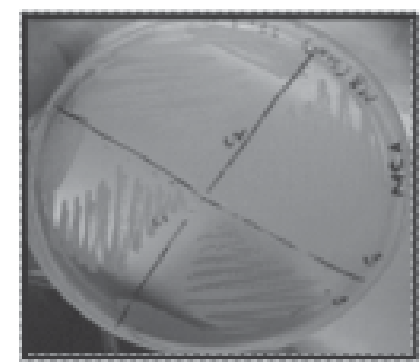

B

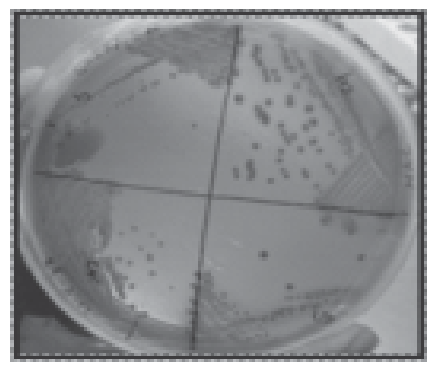

C

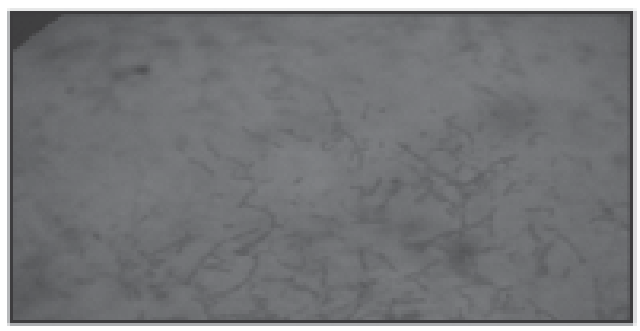

$\mathrm{E}$

Figure 2. Screening procedure of pathogenic microorganisms responsible for mastitis in buffalo. (A) Nutrient agar plate showing different types of colonies representing different organisms; (B) Mannitol salt agar plate: yellow colonies showing growth of mannitol fermenting Staphylococcus aureus whereas white colonies show mannitol non-fermenting other species of Staphylococcus; (C) MacConkey agar plate: Pink colonies showing lactose fermenting E. coli; (D) Gram stained MSA isolates appeared as cocci in clusters and retained violet colour after Gram reaction which represented that they were Gram positive cocci and (E) Gram stained Mac Conkey isolates; appeared rod shaped in chains and retained red colour after Gram reaction indicating Gram negative bacillus. 


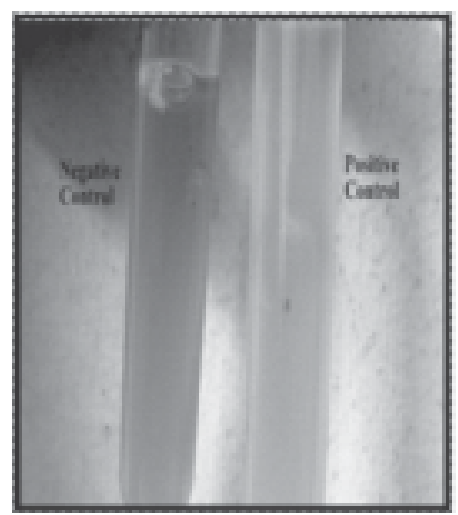

A

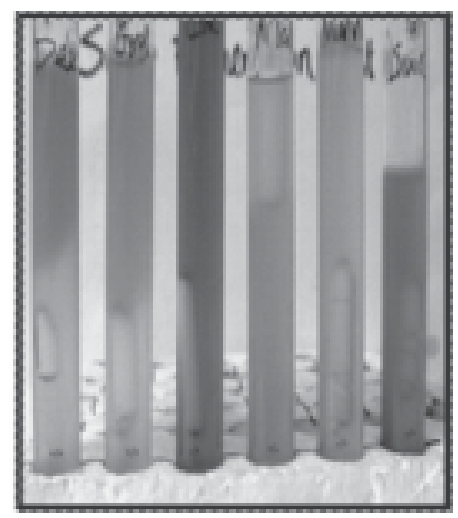

B

Figure 3. Results of various sugar fermentation test. (A) represents positive and negative control, (B) represents different sugar fermenting reactions and gas production, and $(C)$ represents results of various fermentation reaction. Positive strains were seen to ferment sugars turning the color of the indicator from purple to yellow and negative strains were found to be non-fermenters and that is why retaining the colour of the indicator. Here, Suc: sucrose; Dex: dextrose; Gal: galactose; Lac: lactose; Mal: maltose; Man: D-mannitol. Here, +/+, fermentation and gas formation;+/-, fermentation and no gas production; -/+, no fermentation and gas production; ---, no fermentation and no gas production.

\begin{tabular}{lccccccc}
\hline Man & Dex & Gal & Lac & Mal & Suc & Presumptive organisms & Total isolates \\
\hline$+/-$ & $+/-$ & $+/-$ & $+/-$ & $+/-$ & $+/-$ & S. aureus & 21 \\
$+/+$ & $+/+$ & $-/-$ & $+/+$ & $+/+$ & $+/+$ & E. coli & 12 \\
$-/-$ & $+/+$ & $+/+$ & $-/-$ & $+/+$ & $+/+$ & Proteus & 1 \\
$-/-$ & $+/-$ & $+/-$ & $+/-$ & $+/-$ & $+/+$ & Bacillus & 2 \\
$+/+$ & $+/+$ & $+/+$ & $+/+$ & $+/+$ & $+/+$ & Enterobacter & 6 \\
\hline
\end{tabular}

\section{Identification of bacterial flora from milk sample}

A total of 42 isolates were collected from the milk samples and selected for primary screening. Biochemical tests revealed that Staphylococcusspp. Was the dominant species from the samples in this study which constitutedup to $50 \%$ of the total bacteria isolated (Figure 4B). Other than Staphylococcus spp., E. coli constituted $28.57 \%$ of the total percentage. Besides, Enterobacter spp. (14.29\%), Bacillus spp. (4.76\%) and Proteus spp. (2.38\%) were also found.

Antimicrobial susceptibility of the isolates identified from the milk sample

Standard antibiotic disc diffusion assay known as Kirby-Bauer disc diffusion assay was applied for antimicrobial susceptibility test. Total ten antibiotic discs were used in this study namely amoxicillin (AML), azithromycin (AZM), chloramphenicol (CHL), ciprofloxacin (CIP), ceftriaxone (CRO), erythromycin (ERM), gentamycin (GNT), levofloxacin (LEV), nitrofurantoine (NFT) and thrimethoprim (SXT) are shown Table 1.Resistance with varying frequencieswas found against all ten antibiotics used. The highest and the lowest resistance were observed against amoxicillin (21.43\%) and chloramphenicol and ciprofloxac in (4.76\%), respectively. The graphical representation of antibiotic resistant patterns was shown in Figure 5

Mastitis is a global problem as it adversely affects animal health, quality of milk and economics of milk production and every country including developed ones suffer huge financial losses ${ }^{8}$. The majority of mastitis cases may be subclinical caused by contagious organism such as Staphylococcus spp. or by environmental organisms such as E.coli. The present study was designed to determine the prevalence of bubaline (dairy buffaloes) mastitis in selected rural dairying areas of Bangladesh and to determine the association of important potential pathogens mainly Staphylococcus spp. and E. coli, with mastitis.

A total of 30 lactation buffaloes were investigated using clinical or under screening CMT during the study period, none of them were found with clinical mastitis and only 21 (70\%) were found with subclinical mastitis. Among the isolated bacterial population, Staphylococcus spp.(50\%) was found to be the most prevalent bacteria compared to the other bacteria in the current study which also supports previous reports ${ }^{9-10}$. The high prevalence of Staphylococcus spp., associated with subclinical mastitis may be due to in appropriate management. This was followed by 

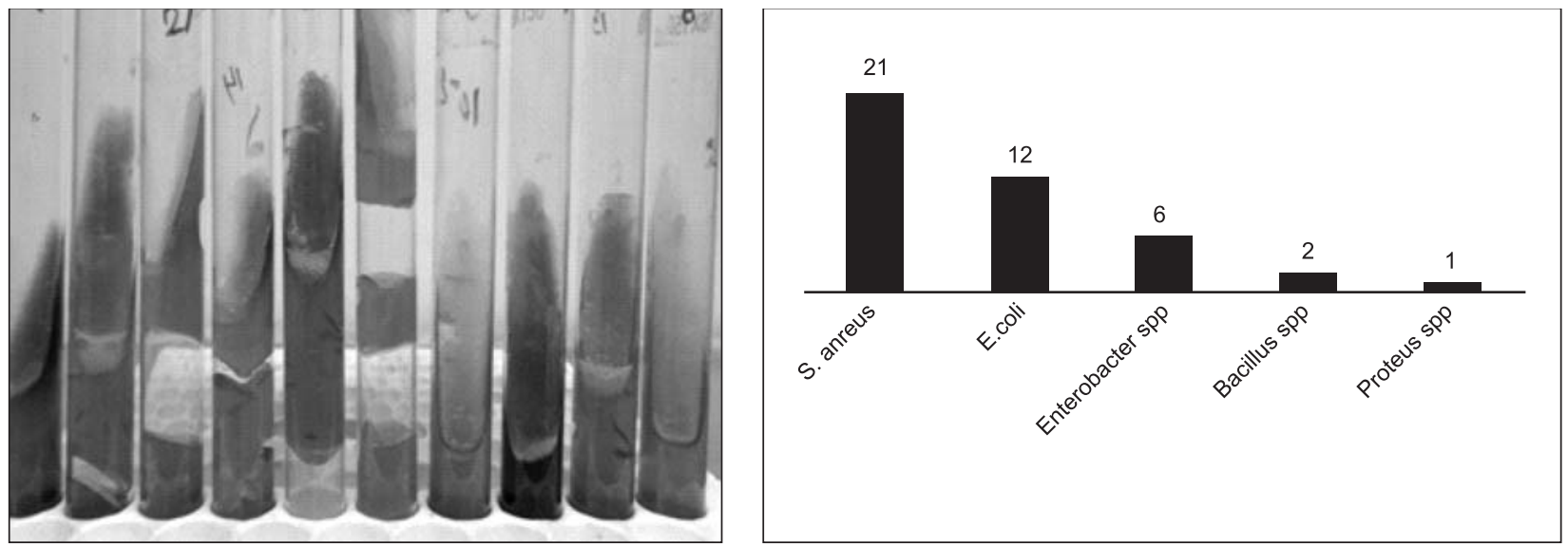

Figure 4. Biochemical characterization of pathogen responsible for mastitis in buffalo. (A) KIA test results showing different reactions in butt and slant accompanied with or without production of gas. A yellow slant or butt indicated the fermentation of glucose or lactose to acidic end products. A red slant or butt indicated that no fermentation of glucose or lactose to acidic end products took place. Cracks or lifting of media or bubbling of media indicated the production of gases other than hydrogen sulfide. (B) Prevalenceof pathogens in mastitis in buffaloes.

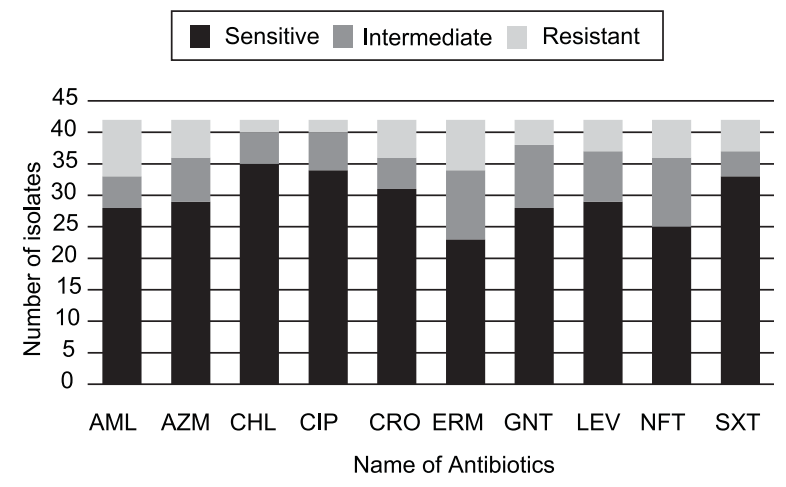

Figure 5. Drug resistant pattern of the pathogenic isolates. $(A M L=$ Amoxicillin,$\quad A Z M=$ Azithromycin,$\quad C H L=$ Chloramphenicol, $C I P=$ Ciprofloxacin, $C R O=$ Ceftriaxone, $E R M=$ Erythromycin, GNT=Gentamycin, LEV=Levofloxacin, NFT=Nitrofurantoine,SXT = Trimethoprim).

E. coli (28.57\%), Enterobacter spp (14.29\%), Bacillus spp. (4.76\%) and Proteus spp. (2.38\%). However the high prevalence of coliforms (Escherichia, Enterobacter) and Bacillus spp. can be attributed to contamination of milk containers from the environment due to the poor hygiene in handling milk along the collection and marketing chain, including the various containers used.

In the present study, only 42 isolates were subjected to antimicrobial susceptibility test against 10 different antibiotics. Among the isolates were tested here, the resistance against chloramphenicol, ciprofloxacin and trimethoprim were less and can be usedin the treatment. Under the conditionwe employed here, $78-83 \%$ isolates were susceptible to chloramphenicol, ciprofloxacin and trimethoprim (Table 1). On the contrary, the majority of the resistances were observed against amoxicillin and erythromycin. This can be attributed to frequent use of these drugs in the selected areas as suggested previously ${ }^{11}$.
Table 1. Antibiotic resistant patterns of pathogens responsible for mastitis in buffalo.

\begin{tabular}{lcc}
\hline Antibiotic & Sensitivity (\%) & Resistance (\%) \\
\hline Amoxicillin (AML) & 66.67 & 21.43 \\
Azithromycin (AZM) & 69.05 & 14.29 \\
Chloramphenicol(CHL) & 83.33 & 4.76 \\
Ciprofloxacin (CIP) & 80.95 & 4.76 \\
Ceftriaxone(CRO) & 73.81 & 14.29 \\
Erythromycin(ERM) & 54.76 & 19.05 \\
Gentamycin(GNT) & 66.67 & 9.52 \\
Levofloxacin(LEV) & 69.05 & 11.90 \\
Nitrofurantoine (NFT) & 59.52 & 14.29 \\
Thrimethoprim(SXT) & 78.57 & 11.90 \\
\hline
\end{tabular}

\section{Conclusion}

In this study, we have identified some pathogenic microorganisms responsible for mastitis in buffalo. Among them, Staphylococcus spp. isolates were most susceptible to ceftriaxone, trimethoprim, chloramphenicol, gentamycin, levofloxacin and amoxicillin; and resistant to amoxicillin, erythromycin and azithromycin (Table 1). Similarly E. coli was highly resistant to ceftriaxone, trimethoprim, azithromycin and nitrofurantoine but susceptible to ciprofloxacin, azithromycin, trimethoprim, chloramphenicol and levofloxacin due to its infrequent usage (Figure 5). Our results are consistent with the result published recently ${ }^{12}$. However, some risk factors should be taken into account for prevention of mastitis in buffalo ${ }^{13}$. Moreover, the etiological agent of mastitis varies from region to region. Some drawbacks of our study are that we did the identification of the microorganisms on the basis of morphological and biochemical tests and the sample size in number were too small. So, for future research, sample size should be increased and additional test including WST, CMT and SCC should be done in addition to genetic identification on the basis of PCR of 
16s rRNA gene of the microorganisms along with some other genetic characterization such as presence of virulence andantibiotic resistance genes should be included.

\section{Acknowledgement}

The authors wish to thank Ministry of Education (17/10, M-15/ 2007/226), Bangladesh for partially funding of this research work.

\section{References}

1. Saadullah M.2012. Buffalo production and constraints in Bangladesh. J. Anml. Plant sci. 22: 221-224.

2. Johan B. 2002. Tropical Ecology and Environmental Management BI0380, 2001/2002. Project Report: Water Buffaloes- Ecology and Usefulness in Africa.

3. Latif MA.1994. Buffalo production in Bangladesh: Problems and prospects. Proceedings of the $1^{\text {st }}$ Asian Buffalo Association Congress, January 17-21, KhonKaen, Thailand.

4. Wellenberg GJ, van der Poel WH, Van Oirschot JT. 2002.Viral infections and bovine mastitis. A Rev Vetern Microbiol. 88: 27-45.

5. Suriyasathaporn W, Heuer C, Noordhuizen-Stassen EN, Schukken YH. 2000. Hyperketonemia and the impairment of udder defense. A Rev Vetern Res. 31: 397-412.

6. Quinn PJ, Carter ME, Markey BK, Carter GR.2002.Clin Vetern Microbiol Clinical and Veternary Microbiology, pp. 311-344. Har Court Publishers, Virginia, USA.
7. Clinical and laboratory Standard Institute. 2010. Performance Standards for Antimicrobial Susceptibility Testing; Twenty-First Informational Supplement. Clin Lab Std Inst.CLSI document M100S20 Wayne PA.

8. Sharma N, Maiti SK, Sharma KK (2007). Prevalence, etiology and antibiogram of microorganisms associated with sub clinical mastitis in buffaloes in Durg, Chhattisgarh State (India). Int J Dairy Sci. 2: 145-151.

9. Kudinha T, Simango C.2002. Prevalence of coagulase-negative Staphylococci in bovine mastitis in Zimbabwe. South Afri Vetern Asson. 73: 62-65.

10. Suarzez VH, Busetti MR, Miranda AO, Calvinho LF, BedottiDO, Canovesio VR.2002.Effect of infectious status and parity on somatic cell count and CMT in Pampinta dairy ewes. Inf Dis Vetern Pub Hel. 49: $230-234$.

11. Jaims EC, Montros LE, Renata DC.2002. Epidemiology of drug resistance; the case of Staphylococcus aureusand Coagulase negative Staphylococci infections. Epid of Drug Resist. 44: 108-112.

12. Srinivasan P, Jagadeswaran D, Manoharan R, Giri T, Balasubramaniam GA, Balachandran P.2013.Prevalence and etiology of subclinical mastitis among buffaloes inNamakkal, India. Pak J. Biol Sci. 16: 1776-1780.

13. Hussain R, Muhammad Tariq Javed, Ahrar Khan and Ghulam Muhammad 2013.Risks factors associated with subclinical mastitis in water buffaloes in Pakistan. Trop Animl Health Prod 45: 1723-1729. 